\title{
Gene Copy Number and Cell Cycle Arrest
}

\author{
Bhaswar Ghosh and Indrani Bose*
}

28th October 2018

\author{
Department of Physics \\ Bose Institute \\ 93/1, A. P. C. Road \\ Kolkata - 700 009, India
}

*Author to be contacted for correspondence; e-mail: indrani@bosemain.boseinst.ac.in Communicated to Physical Biology

\begin{abstract}
The cell cycle is an orderly sequence of events which ultimately lead to the division of a single cell into two daughter cells. In the case of DNA damage by radiation or chemicals, the damage checkpoints in the $G_{1}$ and $G_{2}$ phases of the cell cycle are activated. This results in an arrest of the cell cycle so that the DNA damage can be repaired. Once this is done, the cell continues with its usual cycle of activity. We study a mathematical model of the DNA damage checkpoint in the $G_{2}$ phase which arrests the transition from the $G_{2}$ to the $M$ (mitotic) phase of the cell cycle. The tumor suppressor protein p53 plays a key role in activating the pathways leading to cell cycle arrest in mammalian systems. If the DNA damage is severe, the p53 proteins activate other pathways which bring about apoptosis, i.e., programmed cell death. Loss of the p53 gene results in the proliferation of cells containing damaged DNA, i.e., in the growth of tumors which may ultimately become cancerous. There is some recent experimental evidence which suggests that the mutation of a single copy of the p53 gene (in the normal cell each gene has two identical copies) is sufficient to trigger the formation of tumors. We study the effect of reducing the gene copy number of the p53 and two other genes on cell cycle arrest and obtain results consistent with experimental observations.
\end{abstract}

\section{Introduction}

The cell cycle involves the essential mechanism by which a single cell divides into two daughter cells. Cell division provides the basis for the growth and development of complex organisms and is required to replace cells that die with new cells. The cell cycle is a sequence of orderly events during which a growing cell duplicates all its components so 
cell. In an eukaryotic organism, the cell cycle progresses through four distinct phases: $G_{1}, \mathrm{~S}, G_{2}$ and $\mathrm{M}$ (mitotic) phases [1]. During the S phase, the chromosomes containing DNA molecules are replicated. During the M phase, the sister chromatids are separated and cell division takes place. The $G_{1}$ and $G_{2}$ phases are the gap phases in which cell growth and doubling of the cellular constituents, other than DNA, take place. Cellular growth is necessary so that the daughter cells acquire the same size as that of the parent cell. The cell is arrested at checkpoints if the processes occurring in the earlier stages of the cycle remain incomplete. In the case of DNA damage by radiation or chemicals, the cell cycle is arrested at the damage checkpoints in $G_{1}$ and $G_{2}$. The delay is needed for the repair of the damaged DNA. Once this is done, progression through the cell cycle is resumed once more.

The eukaryotic cell operates through the sequential activation and deactivation of cyclin-dependent protein kinases (CDKs) [1]. Protein kinases are enzymes which regulate the structure and/or activity of target proteins by the transfer of phosphate molecules (phosphorylation). Similarly, protein phosphatases regulate biochemical activity by removing phosphate molecules from target proteins (dephosphorylation). CDK activation can occur only after a regulatory protein cyclin binds CDK and the complex is phosphorylated by CDK-activating kinases (CAKs). Even if these conditions are met, the CDK may be inactivated by inhibitory phosphorylations carried out by certain protein kinases. Protein phosphatases remove the inhibitory phosphate molecules so that the activity of the cyclin-CDK complex is triggered. A cycle of synthesis and degradation of cyclins in each cell cycle controls the periodic assembly and activation of the cyclin-CDK complexes. In higher eukaryotic organisms, different cyclin-CDK complexes initiate different cell cycle events. The cell control system, however, operates on similar principles which may thus be assumed to be universal. In this paper, we focus attention on the $G_{2} / M$ transition in frog egg and mammalian cell cycles. In both the cases, the kinase Wee1 is responsible for the inhibitory phosphorylation of the cyclin-CDK complex and the phosphatase Cdc25 removes the inhibitory phosphate groups [2]. The active cyclinCDK complex phosphorylates key intracellular proteins which in turn initiate or control important cell cycle events. The network of molecular interactions, controlling the stability and activity of the cyclin-CDK complex is characterized by the presence of feedback loops [3]. The loops originate as the proteins (the kinases, phosphatases etc.) influencing the activity of the cyclin-CDK complex are in turn regulated by the activity of the cyclin-CDK complex itself. Mathematical models of the cell cycle have established that the $G_{2} / M$ transition is analogous to a bistable switch $[1,2,3,4,5]$. The bistability arises because the cyclin-CDK complex inactivates its antagonist the kinase Wee1 and activates its friend $\mathrm{Cdc} 25 \mathrm{C}$, giving rise to positive feedback loops. The two stable steady states belong to the $G_{2}$ (low activity of the cyclin-CDK complex) and the $M$ (high activity of the cyclin-CDK complex) phases respectively. The transition between the two stable steady states is not reversible but is described by a hysteresis loop. The transition from the lower to the upper state occurs when the cyclin threshold crosses a critical value. Since the amount of cyclin is correlated with the cell size or cell mass/DNA, the latter quantity can be treated as the parameter the changing of which triggers the $G_{2} / M$ tran- 
confirming earlier theoretical predictions [6].

We now discuss the function of the DNA damage checkpoint in $G_{2}$ which prevents the entry into the $M$ phase when DNAs are damaged [1,7]. The damage induced signal to a series of protein kinases leads to the phosphorylation and inactivation of the phosphatase Cdc25C. Dephosphorylation and consequent activation of the cyclin-CDK complex by $\mathrm{Cdc} 25 \mathrm{C}$ are thus inhibited blocking the transition into mitosis. After the damage is repaired, the DNA damage induced signal is turned off so that the $G_{2} / M$ transition is possible. In terms of the hysteresis loop, the cell cycle arrest corresponds to a higher critical value of the parameter (the cell mass/DNA or analogously the cyclin concentration) at which the $G_{2} / M$ transition takes place. The delay in the transition implies an arrest of the cell cycle progression. In mammalian cells, DNA damage leads to the activation of the tumor suppressor protein p53 which stimulates the transcription of many other genes. One of these genes synthesizes the protein p21 which binds to the cyclin-CDK complex and inhibits its activity. Similarly, DNA damage activates the chk1 protein which has an inhibiting effect on Cdc25c activity through phosphorylation. All these processes give rise to cell cycle arrest allowing for the repair of the DNA damage. If the p53 gene is mutated, there is a reduction in the amount of proteins synthesized so that an arrest of the cell cycle may not occur. As a consequence, the cells containing damaged DNA proliferate through successive rounds of cell division giving rise to the formation and growth of tumors. Such cells pose a threat to the organism as they have a greater probability of becoming cancerous [7]. In fact, many cases of human cancer are attributed to mutations in the p53 gene. The p53 protein, by preventing the multiplication of damaged or stressed cells, acts as a break on the tumor development, hence the name tumor suppressor. Knudson's well-known two-hit model of tumorigenesis suggests that mutation of both the copies (diploid organisms have two copies of each gene) of a tumor suppressor gene is essential for triggering tumor formation $[8,9,10]$. Recent studies, however, show that the mutation of a single copy is sufficient in many cases for the loss of the tumor suppression function of the p53 protein. The gene dosage effect is called haploinsufficiency (HI) and has been verified experimentally [11,12]. Tumors are found to arise in mice with only one intact copy of the p53 gene contrary to Knudson's hypothesis.

In this paper, we propose a mathematical model of the DNA damage checkpoint in the $G_{2}$ phase of the cell cycle to illustrate the effect of gene copy number, i.e., gene dosage on cell cycle arrest. On DNA damage, the $G_{2} / M$ transition is shown to be arrested when the gene copy number is two. When the gene copy number is one, the $G_{2} / M$ transition is not arrested, an effect of HI.

\section{Model of the $G_{2} / M$ transition}

We consider a mammalian system in which the kinase Wee1 inhibits the activity of the cyclin-CDK complex and the phosphatase Cdc25C activates the complex through dephosphorylation. On the other hand, the cyclin-CDK complex in its active state acts as a kinase and inactivates Wee1 through phosphorylation. The complex further activates Cdc25C via phosphorylation. The molecular interactions of the cyclin-CDK complex with 
actions. Reaction schemes in (1) and (2) and the associated differential equations in (3) and (4) lead to the following set of differential equations describing the network shown in figure 1:

$$
\begin{gathered}
\frac{d x_{1}}{d t}=j_{c} m-k_{1} y \frac{x_{1}}{j_{1}+x_{1}}+k z \frac{x_{2}}{j_{2}+x_{2}}-\gamma_{c 1} x_{1} \\
\frac{d x_{2}}{d t}=k_{1} y \frac{x_{1}}{j_{1}+x_{1}}-k z \frac{x_{2}}{j_{2}+x_{2}}-\gamma_{c 2} x_{2} \\
\frac{d y}{d t}=k_{2} \frac{1-y}{j_{3}+1-y}-k_{2}^{\prime} x_{1} \frac{y}{j_{4}+y} \\
\frac{d z}{d t}=k_{3} x_{1} \frac{z_{1}}{j_{5}+z_{1}}-k_{3}^{\prime} \frac{z}{j_{6}+z}-\beta_{1} z \\
\frac{d z_{1}}{d t}=\alpha-k_{3} x_{1} \frac{z_{1}}{j_{5}+z_{1}}+k_{3}^{\prime} \frac{z}{j_{6}+z}-\beta_{1} z_{1}
\end{gathered}
$$

where $x_{1}\left(x_{2}\right)$ denotes the concentration of the active (inactive) cyclin-CDK complex, $y$ is the concentration of the active Wee1 and $z\left(z_{1}\right)$ denotes the concentration of the active (inactive) Cdc25C. The total concentration of Wee1 is normalized to one so that $1-y$ represents the concentration of the inactive Wee1. The $j_{i}$ 's $(i=1, \ldots, 6)$ are the Michaelis-Menten constants. The first term in equation (5) arises due to the synthesis of cyclins, the rate of which is proportional to the cell mass/DNA m. In the $G_{2}$ phase, cells are growing and larger cells, it is assumed, synthesize cyclin at a higher rate [13]. The steady state solutions of equations (5)-(9) are obtained with the help of Mathematica. Figure 3 shows the result in the form of a hysteresis loop with $m$ playing the role of the bifurcation parameter. In the region of bistability, the two stable steady states correspond to the $G_{2}$ (lower branch) and $M$ phases respectively. When $m$ and correspondingly the cyclin concentration in the cyclin-CDK complex reaches a threshold value, the active complex inactivates Wee1 and activates Cdc25C in sufficient amounts. This triggers the autocatalytic conversion of the inactive cyclin-CDK complex into the active complex and a transition to the mitotic phase, with a higher concentration of the active cyclinCDK complex, takes place. Results shown in figure 3 have been obtain for the following parameter values. The rate constants are (in units of $\mathrm{min}^{-1}$ )

$j_{c}=0.02, k=0.01, k_{1}=0.01, \gamma_{c 1}=0.01, \gamma_{c 2}=0.01, k_{2}=0.004, k_{2}^{\prime}=0.2, k_{3}=0.004$, $k_{3}^{\prime}=0.001, \alpha=0.01, \beta_{1}=0.002$.

The Michaelis-Menten constants (dimensionless) are:

$j_{1}=0.5, j_{2}=0.5, j_{3}=0.02, j_{4}=0.02, j_{5}=0.02, j_{6}=0.02$.

Values of the different rate and Michaelis-Menten constants are consistent with those reported in earlier literature $[4,5,13]$ and the forms of the differential equations (equations (5)-(8)) are similar. Bistability and the hysteresis loop are obtained for a wide range of parameter values and changes in the structure of the differential equations, like replacing the Michaelis-Menten kinetics by the mass action kinetics, do not alter the basic result. In the next section, we propose a mathematical model of the $G_{2}$ damage checkpoint which, 


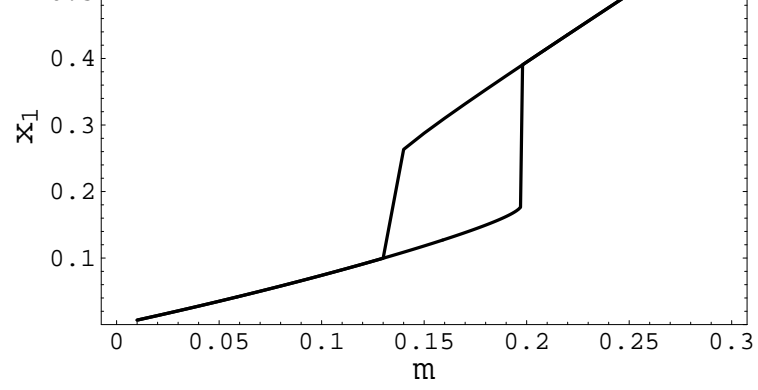

Figure 3. Hysteresis loop for the $G_{2} / M$ transition; $x_{1}$ is the concentration of the active cyclin-CDK complex and $m$ the cellular mass/DNA

combined with the model of this section, predicts a cell cycle arrest on DNA damage. Progression of the cell cycle, however, is not halted if the p53 gene copy number is one instead of two.

\section{DNA damage and cell cycle arrest}

We now discuss how the p53 network is activated on DNA damage in the $G_{2}$ phase of the cell cycle. In a normal cell, the level of the p53 protein is low [7]. The amount depends mostly on the degradation rate of the protein rather than on its rate of synthesis. The p53 protein stimulates the transcription of the MDM2 gene and the MDM2 protein binding to the p53 protein activates its degradation through ubiquitin-mediated proteolysis. The interactions between the p53 and the MDM2 proteins give rise to a negative feedback loop which keeps the p53 protein level low. On DNA damage, a protein called ATM kinase is activated which phosphorylates the p53 protein at a specific site and prevents the binding of the MDM2 protein to the p53. This inhibits the MDM2 mediated degradation of the p53 so that the protein stabilizes at a higher level. The protein p53 activates the transcription of the MDM2 gene giving rise to a negative feedback loop. An effective positive feedback loop coexists with the negative feedback loop (figure 4) due to the p53 mediated inhibition of the transport of the cytoplasmic MDM2 proteins into the cell nucleus [14]. Experimental observations suggest that the p53 and MDM2 proteins are degraded mainly in the nucleus [15]. There is also experimental evidence that DNA damage kinases induce an auto-degradation of the MDM2 proteins [15]. Recently, Lahav et al. [16] have performed a single cell experiment on the dynamics of the p53-MDM2 network after DNA damage. The response of the network to the damage is found to be digital in the form of a discrete number of p53 and MDM2 protein pulses. The amplitude of the pulses and the interpulse time interval do not depend on the amount of DNA damage but the number of pulses is determined by the magnitude of the damage. A negative feedback loop can give rise to oscillations if the number of elements in the loop exceeds two or a time delay is included in the feedback process. A simple mathematical model of the p53-MDM2 negative feedback loop includes an intermediary of unknown origin in order to obtain oscillations [17]. A more recent study [14] proposes a mathematical model in which the p53-MDM2 interaction network is based on both negative and pos- 
two feedback loops. The intermediate processes included in the negative feedback loop introduce the time delay required for getting oscillations. In our simplified model, we assume the existence of feedback loops as shown in figure 4 without explicitly taking into account the biochemical events contributing to the repression of MDM2 by p53. Oscillations in our simplified scheme are generated by considering the expression of the MDM2 gene to be a two-step process, i.e, consisting of both transcription and translation. In the earlier modeling studies [14,17], only one step, namely, protein synthesis was taken into account. The two-step GE introduces a time delay in the p53-MDM2 network dynamics leading to oscillations. The p53 pulses further activate the transcription of a gene p21. The p21 proteins inhibit the activity of the cyclin-CDK complex leading to a delay in the $G_{2} / M$ transition, i.e., a cell cycle arrest [7]. As mentioned in the introduction, DNA damage also activates the chk1 protein which inhibits Cdc25C activity. Figure 5 shows the DNA damage response network which arrests the $G_{2} / M$ transition. In figure 5 , the p53 protein activity is determined by the processes depicted in figure 4. Similarly the cyclin-CDK complex and $\mathrm{Cdc} 25 \mathrm{C}$ are parts of the network shown in figure 1 . The differential equations describing the levels of p53 and MDM2 activity (figures 4 and 5) are:

$$
\begin{aligned}
& \frac{d p}{d t}=j_{0}+x_{d} \frac{p_{a}}{j_{1}^{\prime}+p_{a}}-A \frac{p}{j_{2}^{\prime}+p}-\beta_{2} p m_{p}-\beta_{1} p \\
& \frac{d p_{a}}{d t}=-x_{d} \frac{p_{a}}{j_{1}^{\prime}+p_{a}}+A \frac{p}{j_{2}^{\prime}+p}-\beta_{1} p \\
& \frac{d m_{r}}{d t}=s_{m}+j_{m} \frac{k_{a}^{\prime}}{k_{a}^{\prime}+k_{d}^{\prime}}-\gamma_{m} m_{r} \\
& \frac{d m_{p}}{d t}=j_{p} m_{r}-\gamma_{p} m_{p}-k_{1 m}\left(p+p_{a}\right) m_{p}-k_{2 m} A m_{p} \\
& \frac{d m 21}{d t}=j_{21} \frac{k_{a}^{\prime \prime}}{k_{a}^{\prime \prime}+k_{d}^{\prime \prime}}-\gamma_{m} m 21 \\
& \frac{d p 21}{d t}=j_{p 21} m 21-\gamma_{p 21} p 21
\end{aligned}
$$

with

$$
\begin{gathered}
k_{a}^{\prime}=k_{a} \frac{\left\{\left(p+p_{a}\right) / k_{m}\right\}^{4}}{1+\left\{\left(p+p_{a}\right) / k_{m}\right\}^{4}}, \quad k_{d}^{\prime}=k_{d} \\
k_{a}^{\prime \prime}=k_{a} \frac{\left(p_{a} / k_{21}\right)^{4}}{1+\left(p_{a} / k_{21}\right)^{4}}, \quad k_{d}^{\prime \prime}=k_{d}
\end{gathered}
$$

In the equations, $p$ and $p_{a}$ denote the concentrations of the inactive and the active p53, $m_{r}$ and $m_{p}$ are the concentrations of the MDM2 mRNA and protein, $m 21$ and $p 21$ represent the concentrations of the p21 mRNA and protein and $A$ denotes the amount of DNA damage. In equation (10), the first term describes the production of the p53 protein (inactive), the second term corresponds to the dephosphorylation, i.e., inactivation of 


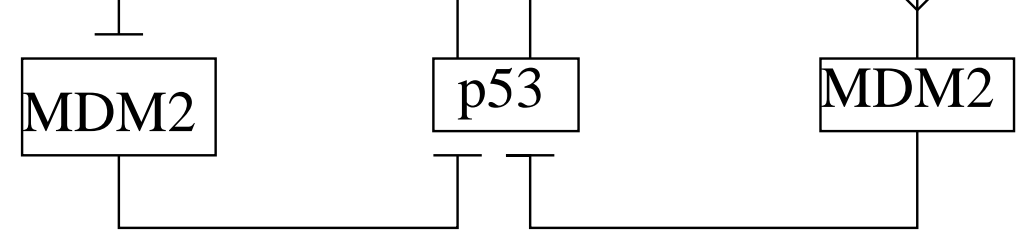

Figure 4. The p53-MDM2 network describing the effective interactions between the p53 and the MDM2 proteins

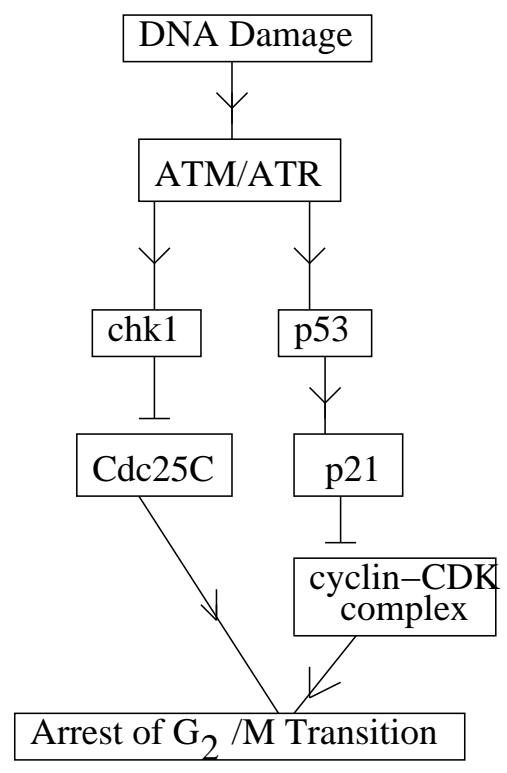

Figure 5. DNA damage response network leading to cell cycle arrest

the active p53, the third term refers to the phosphorylation, i.e., activation of the p53 on DNA damage, the fourth term describes the p53 degradation by the MDM2 protein and the last term refers to the p53 degradation. Equation (12) describes the synthesis of the MDM2 mRNA, the first term of which represents the basal rate of production. The second term denotes mRNA production due to the activation of the MDM2 gene by both the active and inactive forms of p53. The MDM2 gene can be in two states: inactive and active. In the inactive state of the gene, mRNA production takes place at the basal rate $s_{m}$. In the active state of the gene (activation brought about by the transcription factor p53), mRNA production occurs with rate constant $j_{m}$. The p53 proteins tetramerize to regulate the MDM2 gene expression. The reaction scheme describing the production and degradation of mRNA and protein is given by

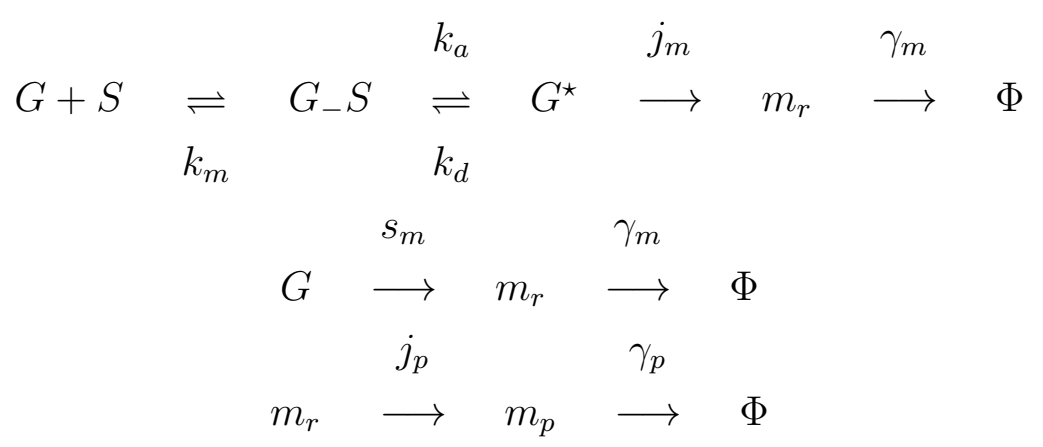

where $G\left(G^{*}\right)$ denotes the inactive (active) state of the gene, $S$ denotes the p53 tetramer, 


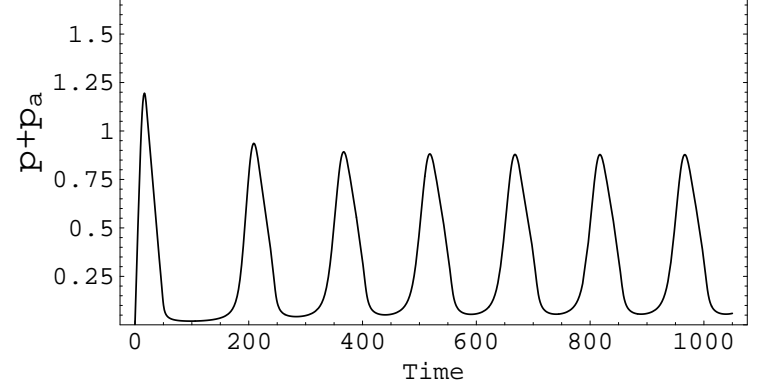

Figure 6. Pulses of p53 proteins generated on DNA damage; $\left(p+p_{a}\right)$ is the total p53 concentration.

$k_{m}$ is the equilibrium dissociation constant, $\gamma_{m}, \gamma_{p}$ the rate constants for mRNA and protein degradation and $k_{a}, k_{d}$, the activation and deactivation rate constants. In equation (11), $k_{a}^{\prime}$ and $k_{d}^{\prime}$ denote the effective rate constants for activation and deactivation and are given in equation (16). In equation (13), the third term takes into account the inhibiting effect of p53 on the MDM2 protein (figure 4) while the fourth term denotes the damage induced degradation of the MDM2 protein [15]. In equation (14), the first term arises due to the activation of the p21 gene by the activated p53 protein. The effective rate constants $k_{a}^{\prime \prime}$ and $k_{d}^{\prime \prime}$ are analogous to the rate constants $k_{a}^{\prime}$ and $k_{d}^{\prime}$ in equation (11) and are given in equation (17). Figure 6 shows the response of the p53 network (figures 4 and 5) to DNA damage. The p53 protein is produced in pulses consistent with experimental observation [16]. The rate constants are (in units of $\mathrm{min}^{-1}$ )

$j_{0}=0.1, x_{d}=0.04, \beta_{2}=0.2, \beta_{1}=0.01, s_{m}=0.0001, j_{m}=1, k_{a}=10, k_{d}=10$, $\gamma_{m}=0.01, j_{p}=2, \gamma_{p}=0.01, k_{1 m}=2, k_{2 m}=0.01, j_{21}=0.4, j_{p 21}=2, \gamma_{p 21}=0.005$

The Michaelis-Menten constants (dimensionless) are

$j_{1}^{\prime}=0.01, j_{2}^{\prime}=2$.

The binding constants $k_{m}$ and $k_{21}$ are $k_{m}=2, k_{21}=1$. The amount of DNA damage is $A=0.2$.

We now study the cell cycle arrest on DNA damage by combining the operations of the cyclin-CDK complex (figure 1) and the DNA damage response network (figures 4 and 5 together). Since the chk1 proteins inhibit the activity of $\mathrm{Cdc} 25 \mathrm{C}$ and the p21 proteins inactivate the active cyclin-CDK complex, the inclusion of these processes in equations (5) and (8) leads to the following modified equations:

$$
\begin{gathered}
\frac{d x_{1}}{d t}=j_{c} m-k_{1} y \frac{x_{1}}{j_{1}+x_{1}}+k z \frac{x_{2}}{j_{2}+x_{2}}-\gamma_{c 1} x_{1}-\delta p 21 x_{1} \\
\frac{d z}{d t}=k_{3} x_{1} \frac{z_{1}}{j_{5}+z_{1}}-k_{3}^{\prime} \frac{z}{j_{6}+z}-\beta_{1} z-k_{4} p_{c h k 1}^{a} z
\end{gathered}
$$

The last terms in equations (19) and (20) take into account the damage induced interactions mentioned above. The time evolution of the concentrations of the inactive $\left(p_{c h k 1}^{i n}\right)$ and the active $\left(p_{c h k 1}^{a}\right) \operatorname{chk} 1$ proteins is given by

$$
\frac{d p_{c h k 1}^{i n}}{d t}=j_{c h k 1}+\omega_{1} \frac{p_{c h k 1}^{a}}{j_{3}^{\prime}+p_{c h k 1}^{a}}-\omega_{2} A \frac{p_{c h k 1}^{i n}}{j_{4}^{\prime}+p_{c h k 1}^{i n}}-\gamma_{c h k 1} p_{c h k 1}^{i n}
$$




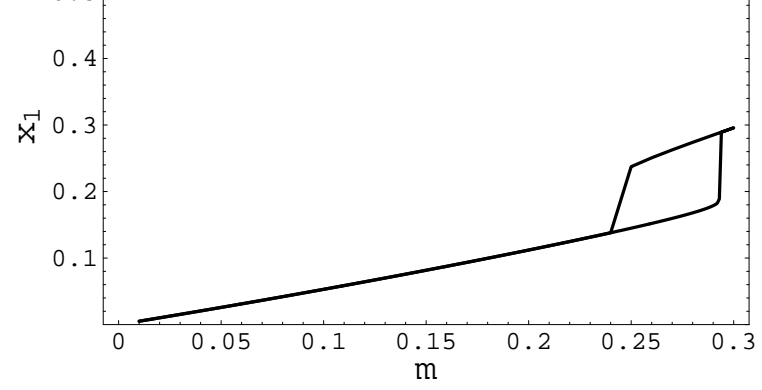

Figure 7. Cell cycle arrest on DNA damage; $x_{1}$ is the concentration of the active cyclinCDK complex and $m$ the cellular mass/DNA

$$
\frac{d p_{c h k 1}^{a}}{d t}=-\omega_{1} \frac{p_{c h k 1}^{a}}{j_{3}^{\prime}+p_{c h k 1}^{a}}+\omega_{2} A \frac{p_{c h k 1}^{i n}}{j_{4}^{\prime}+p_{c h k 1}^{i n}}-\gamma_{c h k 1} p_{c h k 1}^{a}
$$

Figure 7 is obtained for $\delta=0.004, k_{4}=0.1, j_{c h k 1}=0.01 \omega_{1}=0.1, \omega_{2}=2, j_{3}^{\prime}=0.01$, $j_{4}^{\prime}=0.03$ and $\gamma_{c h k 1}=0.01$ by solving the set of differential equations (6), (7), (9), (19), (20), (21), (22) and (10)-(15). The values of the other constants are as already specified. Comparing figure 7 with figure 3, one finds that the the cell cycle is arrested due to a shift in the critical value of the cell mass/DNA, required for the $G_{2} / M$ transition, to a higher value. The delay allows the repair of DNA damage to be undertaken. Figure 7 has been obtained assuming the gene copy number of the p53 gene to be two. We now consider the case when one of the gene copies is mutated. This gives rise to a fall in the amount of proteins synthesized by $50 \%$. The effect of reduced gene copy number is incorporated in the mathematical model by halving the value of the rate constant $j_{0}$ (equation (9)) associated with the synthesis of p53 proteins. Figure 8 shows the hysteresis loop when the gene copy number is reduced to one. Comparison of figures 3 and 8 show that the cell cycle is not arrested and the $G_{2} / M$ transition occurs at the same critical value of the cell mass/DNA as in the case of undamaged DNA.

The failure of the DNA damage response network to arrest the cell cycle when the gene copy number of the p53 gene is one provides a clear demonstration of HI associated with the p53 gene. A question that naturally arises in this context is whether the chk1 and MDM2 genes also exhibit a similar effect. In the case of the chk1 gene, reduced gene copy number implies a diminished production of $p_{c h k 1}$ proteins. The effect is taken into account by replacing $j_{c h k 1}$, the rate constant for protein synthesis, by $j_{c h k 1} / 2$ in equation (21). In this case, one finds that the cell cycle is still arrested with almost the same time delay as in the case when the gene copy number of the chk1 gene is two. An interaction not included in the DNA damage response network of figure 5 is the activation of the p53 protein by the activated chk1 protein [18]. When this interaction is included, the chk1 exhibits HI, i,e., the cell cycle is not arrested when the gene copy number of the chk1 gene is reduced from two to one. In the case of the MDM2 gene, reduction of the gene copy number to one $\left(s_{m}\right.$ and $j_{m}$ in equation (12) replaced by $s_{m} / 2$ and $j_{m} / 2$ ) introduces a larger time delay, i.e., shifts the $G_{2} / M$ transition point to a higher value of $m$ (figure 9 ) but the region of bistability shrinks to a tiny size. With the increase in the amount of DNA damage the hysteretic transition is lost. The small size of the region 
different manifestation of HI. Figure 10 shows the $G_{2} / M$ hysteretic transition when the gene copy number of both the p53 and MDM2 genes is one. In this case, one finds that the cell cycle is arrested though with lesser time delay than in the case when the gene copy number of both the p53 and MDM2 genes is two.

Mendrysa et al. [19] have shown that a reduction of the MDM2 protein level in vivo results in increased radiosensitivity. In our study of the DNA damage checkpoint in $G_{2}$, we assumed the amount of DNA damage to be $A=0.2$. We now consider $A$ to have the magnitude 0.17, i.e., reduced DNA damage. Figure 11 shows the $G_{2} / M$ transition

when both the p53 and MDM2 genes have gene copy number two. In the absence of the DNA damage, the $G_{2} / M$ transition occurs as in figure 3. Comparison of the figures 3 and 11 show that the time delay in the $G_{2} / M$ transition is practically negligible, i.e., the damage response network is insensitive to DNA damage. Figure 12 shows the same transition when the gene copy number of the MDM2 gene is reduced to one. This gives rise to decreased MDM2 protein levels and a time delay in the $G_{2} / M$ transition. In other words, the DNA damage response network becomes more sensitive with reduced MDM2 protein levels. With increased DNA damage, diminished MDM2 protein levels may give rise to abnormal cell cycle features like tiny hysteresis loops (figure 9) or the absence of a region of bistability. These examples indicate an increased damage sensitivity with decreased MDM2 protein levels and may sometimes have lethal consequences for the cellular integrity.

\section{Conclusion and Outlook}

In this paper, we study a mathematical model of the $G_{2} / M$ transition in the mammalian cell division cycle and discuss how this transition is arrested when the cellular DNA is damaged by radiation or chemicals. The $G_{2} / M$ transition is a result of bistability with the two stable steady states associated with the $G_{2}$ and $M$ phases. Bistability arises due to the presence of positive feedback loops in the network involving the cyclin-CDK complex, Wee1 and Cdc25C (figures 1 and 2). Bistability is further accompanied by a hysteresis loop (figure 3) the existence of which has been verified experimentally [6]. The function of the DNA damage response network (figure 5), when activated by damage induced signals, is to halt the progression of the cell cycle, i.e., to arrest the $G_{2} / M$ transition. The arrest introduces a time delay in the transition as a greater amount of cyclin or equivalently cell mass/DNA is required for the transition to occur. A core component of the damage response network is the p53-MDM2 network with effective molecular interactions shown in figure 4. In a normal cell, the p53 protein level is low. On DNA damage, the p53 and MDM2 proteins are produced in pulses (figure 6) consistent with experimental observation [16]. The pathways initiated by the activated p53 and chk1 proteins contribute towards the arrest of the $G_{2} / M$ transition (figure 7 ).

The full mathematical model is described by the set of differential equations (6), (7), (9), (19)-(22) and (10)-(15). The equations describing the network in figure 1 are similar to those in the pioneering studies of Tyson and collaborators [4,5]. The genesis of the effective interactions in the p53-MDM2 network (figure 4) is explained in detail in Ref. 
of transcription and translation in the production of the MDM2 proteins. The simplified mathematical framework is adequate for obtaining p53 and MDM2 oscillations. The modeling of the chk1 and p53 activated pathways in the damage response network of figure 5 is a new contribution. Aguda has earlier carried out a quantitative analysis of the kinetics of the $G_{2}$ DNA damage checkpoint system [20]. His model is more detailed than ours but does not include the experimentally observed features like hysteresis and pulsed production of the p53 and MDM2 proteins. The major focus of our study is to study the effect of gene dosage (gene copy number) on the $G_{2} / M$ transition in the case of DNA damage. To our knowledge, such studies have not been carried out earlier. Borisuk and Tyson [21] have addressed the issue of gene dosage briefly vis-⿳亠㐅冋-vis its effect on limit cycle oscillations describing periodic cell division and have concluded that the period of the limit cycle is in general insensitive to gene dosage (two-fold change in parameter values have been considered). In a diploid organism, each gene exists in two copies and when one of these is mutated, the gene copy number is reduced to one. Consequently, the amount of proteins synthesized is reduced and may fall below a threshold level for the onset of some desired activity. This can give rise to HI, a manifestation of which is in the form of a disease $[22,23]$. A large number of diseases are caused by mutations in genes encoding proteins called transcription factors (TFs). More than 30 different human maladies have been attributed to TF HI. TFs regulate GE by binding at the appropriate region of the DNA. Cooperative interactions among the TFs favour the formation of bound TF complexes (oligomers). Such multimeric complexes are essential for the initiation of GE in many eukaryotic systems. The TFs constitute the stimulus (concentration $S$ ) and the response is quantified by the amount of proteins synthesized (concentration $R$ ) from the target gene. Due to the multimerization of the TFs, the curve $R$ versus $S$ has a sigmoidal shape. A small change in $S$ around the inflection point (the point at which the tangent to the curve has the maximum slope) gives rise to a significant change in the amount $R$ of the response. Thus, if there are two TF encoding genes and one of these is mutated, the level of the TFs produced may fall below the inflection point of the sigmoid leading to a considerable reduction in the amount $R$ of the proteins synthesized from the regulated gene. TF HI occurs if the amount of proteins synthesized falls below a threshold level for the onset of protein activity. In the DNA damage response network (figure 5), the activating p53 proteins tetramerize and act as TFs in regulating the expression of the target genes. Two of these genes are the MDM2 and the p21 genes. The TF activity of the p53 proteins, on DNA damage, initiates processes leading to the cell cycle arrest. The delay in the $G_{2} / M$ transition is desirable to allow time for the repair of the DNA damage. If repair is not possible, the p53 proteins activate other pathways leading to apoptosis, i.e., programmed cell death [1,7]. In the absence of p53 proteins, cell cycle arrest and apoptosis are not possible leading to a proliferation of cells (containing damaged DNA) through successive cell divisions. The unchecked rounds of cell cycle give rise to the formation and growth of tumors which in many cases turn cancerous. Some current studies $[9,10]$ suggest that certain forms of cancer may be a result of TF HI arising from a reduced gene copy number of the p53 tumor suppressor gene. The main result of our study is to show that on DNA damage, the cell cycle is arrested (not arrested) in the $G_{2}$ phase when the 


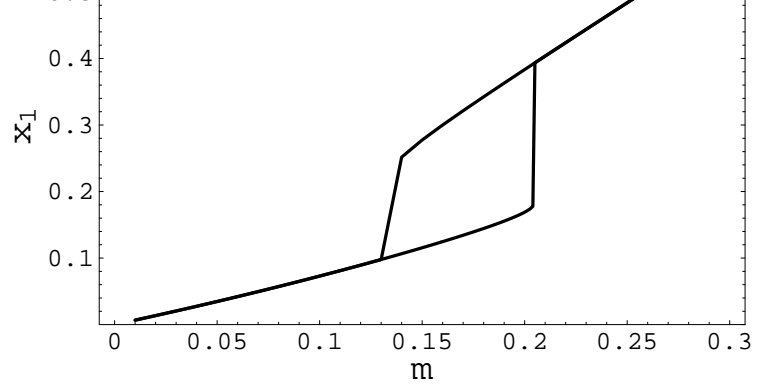

Figure 8. The $G_{2} / M$ transition with one copy of the p53 gene; $x_{1}$ is the concentration of the active cyclin-CDK complex and $m$ the cellular mass/DNA

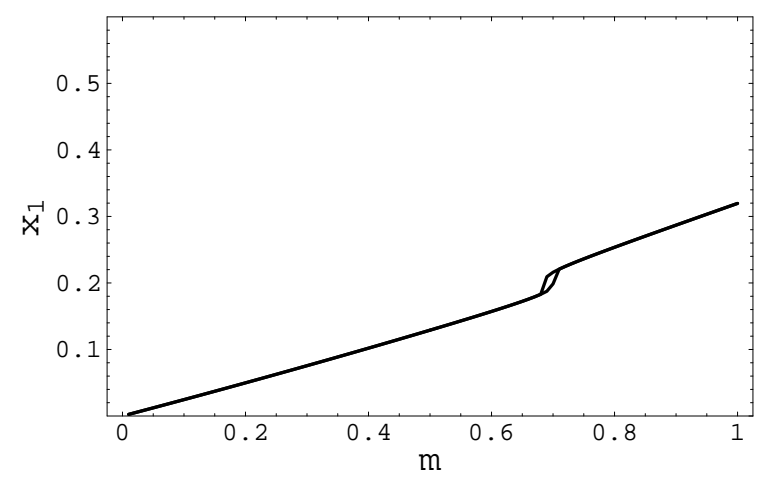

Figure 9. The $G_{2} / M$ transition for one copy of the MDM2 gene and two copies of the p53 gene; $x_{1}$ is the concentration of the active cyclin-CDK complex and $m$ the cellular mass/DNA

gene copy number of the p53 gene is two (one). This is a clear demonstration of HI and one can verify that the result continues to hold true for many other parameter values. We have further explored the issue of whether the chk1 and MDM2 genes also exhibit HI. Our mathematical model predicts observable consequences in both the cases when the gene copy number is reduced to one. The chk1 gene like the p53 gene is a tumor suppressor. The chk1, a protein kinase, is involved in transducing the DNA damage signals and is needed for the operation of the intra-S phase and $G_{2} / M$ checkpoints. Experimental evidence of HI has been obtained in the first case and the possibility of HI in the second case is also not ruled out [24,25]. Our model predicts increased sensitivity of the DNA damage response network when the MDM2 protein levels are reduced which is in agreement with experimental observations [19]. The proposal that HI is responsible for the occurrence of some types of cancer is of recent origin. Experimental evidence on HI related to the DNA damage response of the cell cycle is now beginning to be accumulated. The molecular mechanisms responsible for the control of the damage response are in most cases not fully known. Our preliminary study is meant to highlight the non-trivial effect of gene copy number on the cell cycle response to DNA damage in the $G_{2}$ phase of the mammalian cell cycle. Further studies based on more detailed mathematical models are needed for a clearer understanding of the problem. 


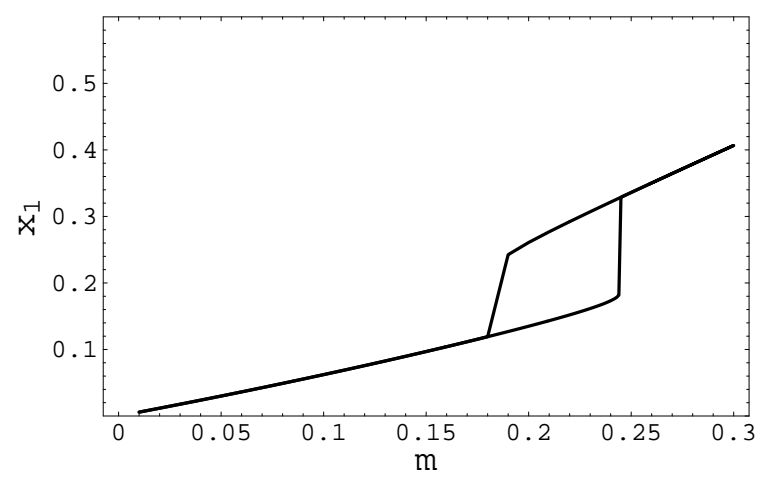

Figure 10. The $G_{2} / M$ transition when the gene copy number of both the p53 and MDM2 genes is one; $x_{1}$ is the concentration of the active cyclin-CDK complex and $m$ the cellular mass/DNA

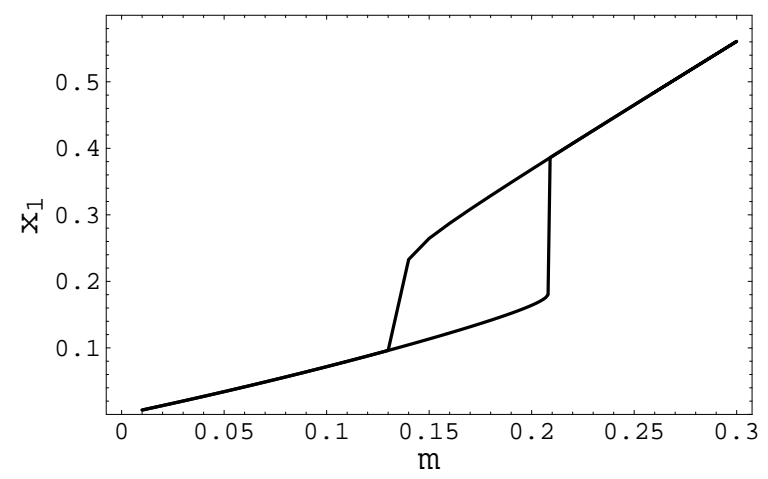

Figure 11. The $G_{2} / M$ transition when the gene copy number of both the p53 and MDM2 genes is two and the amount of DNA damage is $A=0.17$.

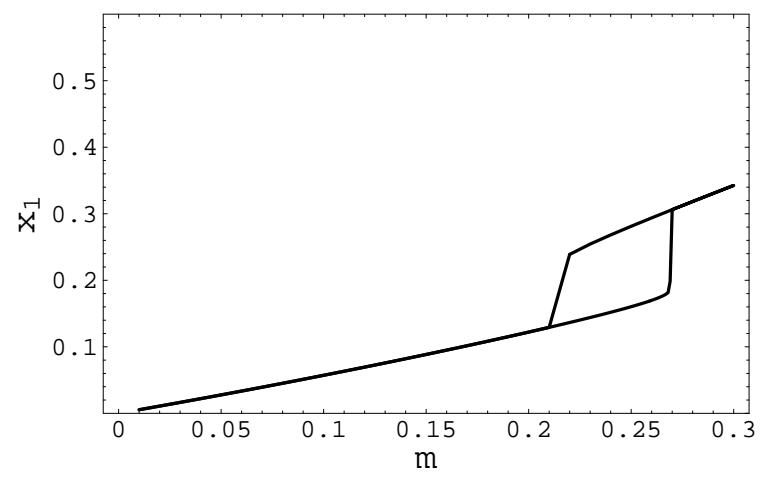

Figure 12. The $G_{2} / M$ transition when the gene copy number of the MDM2 gene is one, that of the p53 gene two and the amount of DNA damage is $A=0.17$ 
B.G. is supported by the Council of Scientific and Industrial Research, India under Sanction No. 9/15 (282)/2003 - EMR-1

\section{References}

[1] Alberts B, Johnson A, Lewis J, Raff M, Roberts K and Walter P 2002 Molecular Biology of the Cell Chapter 17

[2] Tyson J J, Chen K and Novak B 2001 Nat. Rev. Mol. Cell. Biol. 2 908-16

[3] Novak B and Tyson J J 2003 Biochem. Soc. Trans. 31 1526-29

[4] Novak B and Tyson J J 1993 J. Cell Sci. 106 1153-68

[5] Tyson J J and Novak B 2001 J. Theor. Biol. 210 249-63

[6] Sha W, Moore J, Chen K, Lassaletta A D, Yi C-S, Tyson J J and Sible J C 2003 Proc. Natl. Acad. Sci. 100 975-80

[7] Vogelstein B, Lane D and Levine A J 2000 Nature 408 820-823

[8] Knudson A G 1971 Proc. Natl. Acad. Sci. 68 820-23

[9] Fodde R and Smits R 2002 Science 298 761-63

[10] Hohenstein P 2004 PLoS Biology 2 0165-66

[11] Venkatachalam S, Shi Y P, Jones S N, Vogel H, Bradley A and Pinkel D 1998 EMBO J. 17 4657-67

[12] Song W J et. al. 1999 Nature Genet. 23 166-75

[13] Sveiczer A, Csikarz-Nagy A, Gyorffy B, Tyson J J and Novak B 2000 Proc. Natl Acad. Sci. 97 7865-70

[14] Ciliberto A, Novak B and Tyson J J 2005 Cell Cycle 4 107-12

[15] Stommel J M and Wahl G M 2004 EMBO J. 23 1547-56

[16] Lahav G, Rosenfeld N, Segal A, Geva-Zatorsky W, Levine A J, Elowitz M B and Alon U 2004 Nature Genet. 16 147-50

[17] Lev Bar-Or R, Maya R, Segal L A, Alon U, Levine A J and Ures M 2000 Proc. Natl. Acad. Sci 97 11250-55

[18] Kearns W G and Liu J M 2001 Current Genetics 2 171-80

[19] Mendrysa S M, McElwee M K, Michalowski J, O’Leary K A, Young K M and Perry M E 2003 Mol. Cell. Biol. 23 462-73

[20] Aguda B D 1999 Proc. Natl. Acad. Sci. 96 11352-57 
[22] Seidman J G and Seidman C 2002 J. Clin. Invest. 109 451-55

[23] Veitia R A 2002 Bioessays 24 175-84

[24] Lam M H and Rosen J M 2004 Cell Cycle 3 1355-57

[25] Lam M H, Liu Q, Elledge S J and Rosen J M 2004 Cancer Cell 6 45-59 